\title{
Computational Embryology: Past, Present and Future
}

\author{
Sanjeev Kumar and Peter J. Bentley \\ Department of Computer Science, University College London, Gower St., London WC1E \\ 6BT, UK. S.Kumar@cs.ucl.ac.uk, P.Bentley@cs.ucl.ac.uk
}

\begin{abstract}
This chapter describes research into the embryonic field of Computational Embryology. The chapter starts with a brief history of embryology and the contributions scientists have made over the years causing the gradual amalgamation of embryology and genetics to form developmental biology. This is followed by a detailed investigation into the evolution of computational embryogenies. The focus of this chapter is on the two most promising types of embryogeny: Explicit and Implicit, investigating the evolvability and scalability of both embryogenies for morphogenesis. The problem set is that of evolving certain predefined shapes - letters of the alphabet. The results show that both embryogenies are good at defining different morphologies, but significantly, the implicit embryogeny incurs no increase in genotype size as the problem is scaled. Finally, the chapter ends with a description of a more biologically plausible computational model of aspects of biological development.
\end{abstract}

\section{Introduction}

Evolutionary computation (EC) has been a very successful area of computer science for some time. EC has grown from several types of evolutionary algorithms (EAs) which take their inspiration from nature (Bentley, 1999): Genetic Algorithms, Genetic Programming, Evolutionary Strategies and Evolutionary Programming. Although these methods are essentially the same, an important difference is the distinction between the genotype (coded parameters and values) and the phenotype (representation of solutions). GP practitioners often regard the genotype as the phenotype, as do ES and EP practitioners. The genetic algorithm (GA) is the only one of the four EAs that makes the distinction. It is the omission of this crucial genotype to phenotype mapping process, known in biology as an embryogeny, which can often deny the non-GA practitioner the advantages that embryogenies bring.

This chapter investigates the evolvability of two of the most interesting types of computational embryogeny, explicit, and implicit. The chapter is organised as follows: a brief history of embryology is given in section two, section three explains embryology in nature and introduces Computational Embryology. The two embryogeny based systems are introduced in section four, with section five de- 
scribing a series of experiments together with an analysis of the results. Section six introduces a new more biologically plausible model of development. Conclusions are provided in section seven.

\section{From Embryology to Developmental Biology: A Historical Review.}

Man has pondered questions such as his place in the universe and the existence of god for millenia, however a question much closer to home, yet equally baffling is that of development. How are our bodies and other organic entities formed? What gives rise to these bodies? Is the complete body plan of an animal already present in the fertilised egg, i.e. is it preformed? If not, then what mechanisms mould and sculpt such complexity?

Greece saw the formation of such questions starting with Hippocrates, who first addressed the important question of the nature of development, as long ago as the $5^{\text {th }}$ Century BC.

The $4^{\text {th }}$ Century $\mathrm{BC}$ provided the setting to take the question a step further. Aristotle was the first to formulate the problem of how structure arose in the embryo. He proposed two possibilities: Preformationism - namely, that the animal is preformed and simply gets bigger, and the second, Epigenesis (meaning 'upon formation'), namely, that structure arises progressively. Aristotle favoured epigenesis, however in the $4^{\text {th }}$ century religion was prevalent and the church favoured preformationism, which made a hostile environment for epigenesis to compete. Aristotle's preformation/epigenesis dichotomy was to provide the fuel for vigorous debate over the coming centuries.

Some two thousand years later in 1672, Italian scientist Marcello Malpighi provided a detailed description of the chick embryo. Preformationist thinking, endorsed by the church, still prevailed in the $17^{\text {th }}$ century. Unfortunately, Malpighi simply could not help but believe in preformationism, remarking that the embryo was so small that he was just not able to see it, even with his most powerful microscopes.

Fortunately, preformationism was shown to be wrong by the observations of Carl Friedrich Wolff. Wolff did not believe in preformationism and disproved it in 1759 by studying how blood vessels appeared in the chick. Wolff demonstrated that the blood vessels of the chick blastoderm slowly developed from islands of material surrounded by liquid. The reaction to Wolff's evidence was that the blood vessels were there all the time, but became visible later. In 1768, Wolff dealt preformation the final blow, demonstrating that the chick gut was not initially a tube but emerged by the gradual folding of the ventral sheet of the embryo.

The basic unit of life: the cell, was discovered in 1838-1839 by German botanist Matthais Schleiden and physiologist Theodor Schwann. This was a turning point for embryology providing the beginnings of a new era in which embryology was eventually to be subsumed by Developmental Biology.

The mid 1860s saw the Austrian monk and botanist, Gregor Mendel show, by plant hybridization experiments, that the origin of hereditary variability lay in differences in 'factors' (later to be named genes by Johannsen) that pass un- 


\begin{tabular}{|c|c|c|}
\hline Scientist & Date & Contribution \\
\hline Hippocrates & $\begin{array}{c}5^{\text {th }} \text { Century } \\
\text { BC }\end{array}$ & First to address the problem of development. \\
\hline Aristotle & $\begin{array}{l}4^{\text {th }} \text { Century } \\
\text { BC }\end{array}$ & $\begin{array}{l}\text { Aristotle considered two possibilities to the } \\
\text { problem of how structure arose in the } \\
\text { embryo, namely, preformationism and } \\
\text { epigenesis. }\end{array}$ \\
\hline Marcello Malpighi & 1672 & $\begin{array}{l}\text { Provided an accurate description of chick } \\
\text { embryo. }\end{array}$ \\
\hline Carl Friedrich Wolff & $\begin{array}{l}1759- \\
1768\end{array}$ & $\begin{array}{l}\text { Dismissed preformationism by providing } \\
\text { evidence that the chick gut was not initially } \\
\text { a tube but emerged by the gradual folding of } \\
\text { the ventral sheet of the embryo. }\end{array}$ \\
\hline $\begin{array}{l}\text { Mathais Schleiden + } \\
\text { Theodor Schwann }\end{array}$ & $\begin{array}{l}1838- \\
1839\end{array}$ & $\begin{array}{l}\text { Recognised, independently, the cell as the } \\
\text { basic unit of life. }\end{array}$ \\
\hline Gregor Mendel & Mid 1860s & $\begin{array}{l}\text { Proposed, origin of hereditary variability lay } \\
\text { in differences in 'factors' that pass } \\
\text { unchanged from one generation to the next. }\end{array}$ \\
\hline August Weismann & $\begin{array}{l}19^{\text {th }}- \\
\text { End of } 19^{\text {th }} \\
\text { Century }\end{array}$ & $\begin{array}{l}\text { Proposed that the offspring does not inherit } \\
\text { its characteristics from the soma of the } \\
\text { parent, but from the germ cells. } \\
\text { In addition, proposed a 'special factors' } \\
\text { model of development. }\end{array}$ \\
\hline Wilhelm Roux & $\begin{array}{l}\text { Late } \\
1880 \mathrm{~s}\end{array}$ & $\begin{array}{c}\text { Provided confirmation of Weismann's ideas, } \\
\text { by experimenting on frogs. Concluding, frog } \\
\text { development was based on a mosaic } \\
\text { mechanism. }\end{array}$ \\
\hline Hans Driesch & $\begin{array}{l}\text { Late } \\
1880 \mathrm{~s}\end{array}$ & $\begin{array}{l}\text { Disproved Roux's results showing that cells } \\
\text { retain the developmental potential of the } \\
\text { zyogte and were hence not based on a } \\
\text { mosaic mechansim. }\end{array}$ \\
\hline Wilhelm Johannsen & 1909 & $\begin{array}{l}\text { Recognised the distinction between } \\
\text { genotype and phenotype, helping to link } \\
\text { genetics with embryology. }\end{array}$ \\
\hline
\end{tabular}

Table 1: Table showing key contributions in the history of embryology.

changed from one generation to the next.

In the mid $19^{\text {th }}$ century, the German biologist August Weismann proposed that characteristics in the offspring are inherited from the germ cells (the eggs and sperm) and not the soma (body). He further suggested that germ cells were not influenced by the body that bears them. Weismann devised a model of development in which 'special factors' known as determinants were contained in the nucleus of the zygote. These determinants were then unequally distributed to daughter cells during zygote division (cleavage), allowing for control over the 
future development of the cells. In effect, cell fate, according to Weismann's model, is controlled by the unequal distribution of the determinants, and hence predetermined in the egg during cleavage. Weismann's model was termed mosaic, as the egg was likened to a mosaic of discrete localised determinants.

Weismann's ideas received support in the late 1880s due to the German embryologist Wilhelm Roux. Roux provided support by experimenting with frog embryos. After allowing the first cleavage of a zygote, he destroyed one of the two cells with a hot needle. He found that the remaining cells developed into a well-formed half-larva, and concluded that the "development of the frog is based on a mosaic mechanism, the cells having their character and fate determined at each cleavage".

Hans Driesch, also in the late 1880s, disagreed with Roux and set about to disprove Roux's results. Driesch repeated Roux's experiment, but on invertebrate sea urchin eggs, however instead of killing one of the cells at the two-cell stage he separated them. He found that these isolated cells went on to develop normally, concluding that all cells retained the developmental ability of the zygote.

Despite these discoveries it was left to Danish botanist Wilhelm Johannsen in 1909 to not only label Mendel's 'factors' as genes, but also to make the fundamental distinction between genotype (genetic composition) and phenotype (physical appearance). This crucial concept helped link both genetics and embryology giving way to Developmental Biology.

For a more detailed history of embryology, see (Wolpert, 1998) and (Bard, 1990).

\section{Embryology and Embryogenies}

Biology has moved on considerably since Aristotle, and an entire new field now known as Developmental Biology has emerged. Through the centuries of scientific research, we now know that Aristotle's notion of epigenesis was the closest to the truth. Developmental Biology and in particular, Embryology has fast become an exciting and fashionable subject for research.

Today we regard Embryology to be the study of the controlled formation and development of animal and plant embryos. It involves three fundamental processes:

- $\quad$ morphogenesis - which involves the emergence and change of form (Bard, 1990).

- $\quad$ regional specification (pattern formation) - in which compartmentalisation of the embryo into specific regions occurs (Slack, 1991).

- $\quad$ cellular differentiation - in which cells become specialised for particular functions (Wolpert, 1998).

These three processes operate together in different parts of the embryo, at different times, and in stages according to a 'recipe' known as an embryogeny. Embryogenies have evolved in nature to describe how an animal should be grown, rather than contain an overall description of an animal. 
Embryology like other subjects has its own set of problems that need answering. One such problem is that of positional information, i.e. how cells 'know' where to grow? This was addressed by the eminent embryologist Lewis Wolpert who put forth positional information theory (Wolpert, 1998).

Positional information theory states that cells glean their positional information from the free diffusion of a chemical, known as a morphogen, relative to a boundary. The information is coded in the form of the concentration value of the diffusing morphogen. This diffusion sets up a chemical gradient, thus allowing cells to position themselves relative to the boundary whereupon they can, if need be differentiate, i.e., become a specialised type of cell (Wolpert, 1998). The idea of chemical gradients is used in the implicit computational embryogeny described later.

\subsection{Computational Embryology}

Having reviewed biological embryology, this section explores computational embryology.

Computer science first saw the use of morphogenesis in 1952 by Alan Turing. Since then morphogenesis has featured in a number of works, such as in the evolution of neural network morphologies (Jakobi, 1995), and evolvable hardware using cellular encoding (Koza et al. 1999).

However, to date computer science has used crude approximations of the natural embryological processes, paying little attention to the intricate subtleties. Regional specification, which plays an important part in the early stages of embryogenesis has to the authors' knowledge not been investigated, despite its obvious advantages for the creation of form, such as compartmentalisation (which can be viewed as reducing the problem down to smaller units).

Nevertheless, researchers have been using simple computational embryogenies of various guises for over a decade. Current computational embryogenies can be classified into three different types: external, explicit, and implicit (Bentley \& Kumar, 1999).

Most external embryogenies are hand-designed and are defined globally and externally to genotypes. For example, Evolutionary Art systems often use embryogenies defined by fixed, non-evolvable structures which specify how phenotypes should be constructed using the genes in the genotypes (Bentley, 1999). Similarly, Richard Dawkins' Blind Watchmaker program (Dawkins, 1987), employs a simple external embryogeny to create biomorphs, using the "eye of the beholder' to provide a measure of fitness, and mutation to vary the evolving shapes. Dawkins also points out advantages of using embryogenies, why they are so important, and perhaps most interestingly, that different embryogenies can lead to different results. For example, different embryogenies allow different areas of 'solution space' to be searched and thus in doing so constrain themselves to different types of shapes or designs (not necessarily a bad thing as Dawkins shows, relative to what he calls naïve pixel-peppering).

An explicit embryogeny specifies each step of the growth process in the form of explicit instructions. In computer science, an explicit embryogeny can be viewed as a tree containing a single growth instruction at each node. Genetic Pro- 
gramming (GP) uses tree structures to represent its genotypes. GP therefore, offers a simple and concise way to evolve explicit embryogenies. Typically, the genotype and the embryogeny are combined and both are allowed to evolve simultaneously. Examples of explicit embryogenies include Coates (1997) who uses Lindenmayer systems to evolve architectural form. Koza et al (1999) use an explicit embryogeny in the form of cellular encoding for the evolution of analogue circuits. Sims (1999) uses an explicit embryogeny with the idea of directed graphs to specify the nervous systems (neural networks), and morphologies of virtual creatures.

An implicit embryogeny does not explicitly specify each step of the growth process. Instead, the growth process is implicitly specified by a set of rules or instructions, similar to a 'recipe' that govern the growth of a shape. For example, de Garis (1999) describes an implicit embryogeny to evolve convex and concave shapes using a cellular automata approach along with the notion of cellular differentiation. He has reported encouraging results, as well as highlighting problems that need to be tackled in order to improve this approach (de Garis, 1999).

\section{Evolving Embryogenies}

Previous work (Bentley \& Kumar, 1999), compared the performance and scalability of different evolved computational embryogenies for the representation of tessellating tiles. Subsequent experiments have shown that significant questions remain concerning the evolvability of different embryogenies. Specifically, evolved implicit and explicit embryogenies show inconsistent abilities to define specific morphologies. Consequently, it was decided that further investigation was necessary to help explore and understand these issues of evolvability.

\subsection{Evolving Predefined Shapes}

In order to assess the change in performance of the two embryogenies, a number of fixed shapes were specified as targets for evolution. Since shapes with distinct and useful characteristics were desired (e.g. convex, concave, solid, hollow, curved and linear), a subset of the alphabet was selected. Six letters were chosen: $\mathrm{C}, \mathrm{E}, \mathrm{G}, \mathrm{L}, \mathrm{O}$, and $\mathrm{R}$, as shown in figure 1 .
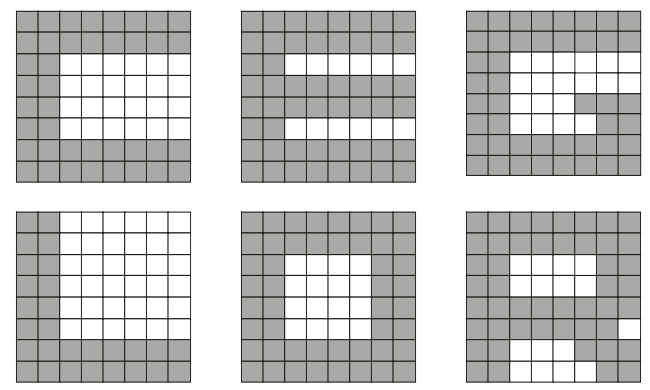

Figure 1: The pre-defined six target shapes. 
These six letters were selected based upon how much of the alphabet they were representative of. For example, the diagonal in the letter $\mathrm{R}$ forming the bottom right portion of the letter is characteristic of the letters $\mathrm{M}, \mathrm{N}, \mathrm{W}, \mathrm{X}, \mathrm{Y}, \mathrm{Z}$. Likewise, the semi-circle is characteristic of the letter P,R,B. The Letters $\mathrm{E}$ and $\mathrm{L}$ with their upright stem are characteristic of P,T,D,F.

To judge how closely each evolving shape matched the targets, a fitness function based on the number of incorrectly filled squares was employed. The fitness score is incremented by one whenever an element in the evolving shape differs from the corresponding element in the current target, see figure 2 . To assess scalability, three different phenotype grid sizes of $4 \times 4,8 \times 8$ and $16 \times 16$ cells were used.

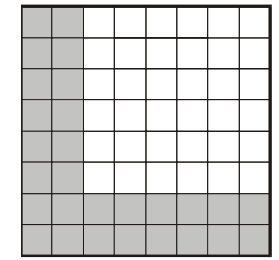

[a]

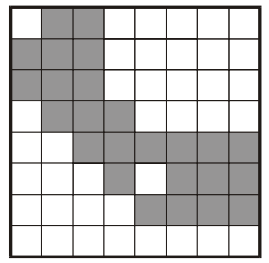

[b]

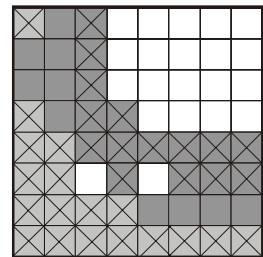

[c]

Figure 2: Calculating the fitness of an $8 \times 8$ evolving shape. [a] shows the target, [b] shows the shape to be judged, [c] shows the incorrect elements identified by the fitness function.

\subsection{Explicit}

This first system used genetic programming (GP) (Koza, 1992) to evolve explicit embryogenies in the form of program trees. Beginning at a seed or zygote cell placed in the phenotype grid, the embryogeny defines the direction of growth at every point. Four functions were used: LEFT, RIGHT, UP and DOWN, with each node in the tree allowed up to four branches. Paths of growth were permitted to overlap. Figure 3 shows an example genotype defining the explicit embryogeny. The root node has two parts: $x$ and $y$ for the co-ordinates of the seed.

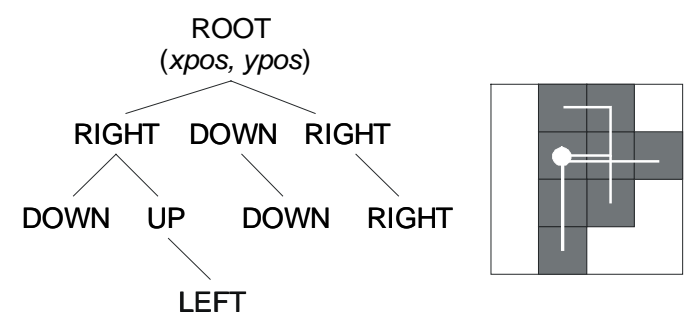

Figure 3: An example explicit embryogeny defined by a tree of nine nodes, and its corresponding $4 \times 4$ phenotype.

The GP system used steady-state selection and a crossover designed to minimise disruption by crossing parents at points of similarity in the two trees. Further details of this system and crossover operator can be found in (Bentley, 1999 and 
Bentley \& Wakefield, 1996). As with all GP systems, bloat occurred, so an additional fitness function penalised genotypes with more nodes. This system differed from the one presented in (Bentley \& Kumar, 1999), in that it evolved the coordinates of the seed.

\subsection{Implicit}

The second system was an advanced variable length chromosome GA that evolved implicit embryogenies. Each genotype comprised a variable number of rules (usually between four and eight). Each rule had a precondition and an action. Each precondition had six fields: LEFT, RIGHT, UP, DOWN, X, Y. A specific rule can take the following values for each precondition field (where \# is don't care, 0 is empty, 1 is filled, $\mathbf{0 , 1 , 2 , 3 , 4 , 5 , 6 , 7}$ are gradient zones):

$\begin{array}{cccccc}\text { LEFT } & \text { RIGHT } & \text { UP } & \text { DOWN } & \text { X } & \text { Y } \\ 0,1, \# & 0,1, \# & 0,1, \# & 0,1, \# & \mathbf{0 - 7}, \# & \mathbf{0 - 7}, \#\end{array}$

For a rule to be fired, values in at least four of the six fields in the precondition must be matched. (This provides the equivalent of disjunction for rule preconditions.) The action of a rule can be: DIE, UPDATE, or grow LEFT, RIGHT, UP or DOWN.

Growth takes place in a phenotype grid, which as usual can be $4 \times 4,8 \times 8$ or $16 \times 16$ elements. In order to permit evolution of specialised rules that can provide detail in specific areas of the phenotype, the grid has two 'gradients' - one in the $\mathrm{x}$ direction, one in the y direction. In a similar way to the gradients used to provide positional information in eggs and wombs of nature (Wolpert, 1998, Slack, 1991, Lawrence, 1995), the gradients divide the grid into eight zones per axis (as opposed to four in previous work), regardless of the number of elements in the phenotype grid.

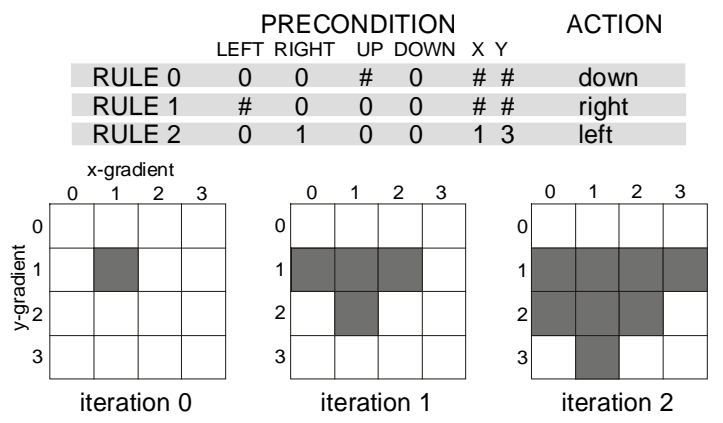

Figure 4: Example of a three-rule implicit embryogeny and its corresponding phenotype after two iterations.

At iteration zero, a seed cell is placed in the phenotype grid at a position defined by the coordinates held in dedicated genes of the chromosome. To model biological cell growth, the rules in the genotype are then applied for a fixed number of iterations to each filled element in the current embryonic phenotype grid. 
(This is unlike traditional cellular automata, where rules are applied to empty or filled grid elements.) Depending on whether the neighbouring elements of the current element exist or not, and on the strength of the two gradients at that point, the rules may be activated, causing growth or cell death in the phenotype. Rules are applied 'in parallel' so that the results of applying the rules to each filled element only take effect at the end of each iteration step. However, a rule which performs the UPDATE action causes all activated rules in the current iteration to be applied. By prematurely placing cells in the phenotype grid in this way, evolution can increase the number of rules applied in each iteration and provide extra growth where needed. This new type of rule action was added to the embryogeny because during the development of the system, the number of iterations was found to be overly critical. Finally, the system was also given the ability to evolve the seed co-ordinates. Figure 4 shows the growth of a target shape, defined by three rules.

\section{Experiments}

\subsection{Objectives and Parameters}

The experimental objectives were three-fold: firstly to investigate the use of both embryogenies for efficiency of search in terms of fitness. Secondly, to investigate the scalability of both embryogenies for evolving different morphologies, and finally, to see how the evolution of the two embryogenies differs in defining different morphologies.

A total of 50 runs were performed with each target shape (letters C, E, G, L, O and R) for each grid size. Population sizes of 100 and a total of 100 generations were used for each run. The explicit embryogeny system used an initial tree depth of 4 for the $4 \times 4,5$ for the $8 \times 8$ and 6 for the $16 \times 16$ grids. All trees were created randomly.

The implicit system used random rule initialisation for both the initial population and for each new rule added to the genome ${ }^{1}$. The cellular automata presented in this work used iteration values of 4 for the $4 \times 4,8$ for the $8 \times 8$, and 14 for the $16 \times 16$ grids. Both systems used random crossover for offspring creation. The explicit system employed a mutation probability rate of 0.001 per bit, whereas preliminary experiments revealed that the implicit system required a rule mutate rate of 0.5 and an increased bit mutation rate of 0.05 .

\footnotetext{
${ }^{1}$ This is as opposed to copying an existing rule as in previous work (Bentley \& Kumar 1999).
} 
Table 2: Results for the target shapes. Values in italics denote the results for the implicit embryogeny. Solution sizes are measured in tree nodes for the explicit, and rules for the implicit embryogeny.

\begin{tabular}{|l|l|l|l|l|l|l|}
\hline \multicolumn{3}{|l}{} & $4 \times 4$ & \multicolumn{2}{l|}{$8 \times 8$} & $16 x 16$ \\
\hline Shape & $\begin{array}{l}\text { Mean } \\
\text { Soln. Size }\end{array}$ & $\begin{array}{l}\text { Mean } \\
\text { Fitness }\end{array}$ & $\begin{array}{l}\text { Mean } \\
\text { Soln. Size }\end{array}$ & $\begin{array}{l}\text { Mean } \\
\text { Fitness }\end{array}$ & $\begin{array}{l}\text { Mean } \\
\text { Soln. Size }\end{array}$ & $\begin{array}{l}\text { Mean } \\
\text { Fitness }\end{array}$ \\
\hline $\mathrm{C}$ & 14.28 & 0.92 & 57.70 & 13.20 & 309.40 & 84.1 \\
& 12.70 & 1.64 & 11.47 & 12.82 & 10.00 & 53.7 \\
\hline $\mathrm{E}$ & 24.22 & 1.28 & 168.44 & 9.54 & 693.58 & 81.40 \\
& 11.42 & 0.32 & 11.96 & 5.89 & 6.700 & 49.40 \\
\hline $\mathrm{G}$ & 18.88 & 1.2 & 59.52 & 12.72 & 302.12 & 76.34 \\
& 13.86 & 0.78 & 10.98 & 12.84 & 6.000 & 52.90 \\
\hline $\mathrm{L}$ & 9.52 & 0.26 & 71.04 & 3.56 & 235.46 & 39.46 \\
& 11.22 & 0.58 & 9.02 & 6.38 & 8.200 & 38.40 \\
\hline $\mathrm{O}$ & 20.20 & 1.31 & 81.29 & 15.76 & 293.00 & 104.33 \\
& 11.60 & 0.18 & 13.29 & 9.00 & 6.400 & 48.70 \\
\hline $\mathrm{R}$ & 18.78 & 1.35 & 121.53 & 7.88 & 513.43 & 76.16 \\
& 12.98 & 0.60 & 12.33 & 9.92 & 6.900 & 55.80 \\
\hline
\end{tabular}

\subsection{Results}

A summary of the results from the experiments is given in Table $2^{2}$. As shown in the table, both embryogenies attained good fitnesses for all $4 \times 4$ target letters. However, relative performances between the two approaches were inconsistent. For example, the explicit embryogeny outperformed the implicit for the letters $\mathrm{C}$ and $\mathrm{L}$, whilst the reverse was true for the other targets.

For the $8 \times 8$ grid, fitness scores were reduced, on average for both methods. For example, the explicit embryogeny managed 3.55 at best for the letter ' $\mathrm{L}$ ' and at worst 15.76 for the ' $\mathrm{O}$ '. The implicit faired similarly on the $8 \times 8$ targets achieving a fitness of 5.89 for the 'E' and only 12.84 for the 'G'. Again, relative performances varied, this time with each embryogeny providing better scores for 3 of the letters.

When the problem was scaled up to the $16 \times 16$ grid, the results show that the implicit embryogeny outperformed the explicit, in terms of fitness. The figures are, however, a little deceptive. For these targets, many of the shapes evolved by the implicit embryogeny were solid blocks. Because of the simple nature of the fitness function, such shapes were awarded higher fitness scores compared to the attempts of the explicit. (Nevertheless, it should be noted that the forms generated by the explicit rarely resembled the desired targets, either.)

Execution times were noticeably different for the two techniques. As the scale of the problem was increased, both methods took longer to grow shapes, but of the two, the implicit required the most computation time. For example, evolution

\footnotetext{
${ }^{2}$ Because of time constraints, average values given for the $16 \times 16$ targets using the implicit embryogeny were based on only 10 experiments per target.
} 
time of six hours for one run of the implicit was not uncommon, compared to less than an hour for the explicit.

Perhaps the most significant results shown in Table 2 are the solution sizes. It is clear that the explicit embryogeny required ever-increasing tree sizes as the scale of the target shapes were increased. However, the reverse seems to be true for the implicit embryogeny, where the number of rules actually appears to decrease as the problems are scaled up. This lack of increase of solution size corroborates and confirms the results obtained in previous work which reported similar findings (Bentley \& Kumar, 1999).

\subsection{Analysis}

The results show interesting behaviours of both embryogenies for all grid sizes. For the $4 \mathrm{x} 4$ grid, because of the size of the targets, the ability of both methods to find good solutions is not surprising. The explicit embryogeny uses small trees to define its solutions, but the implicit often seems to evolve more rules than are necessary. More specifically, a larger number of rules are evolved than are actually used during the growth process. The reason for the inefficiency for such small targets seems to be to do with the search process - it is very hard for evolution to find the correct rules for specific shapes. Clearly the 'add rule' mutation plays an important, but excessive role for these smaller problems. Rules are added until an appropriate collection exists to define the target, but the unused rules are not removed by mutation, much like bloat in GP.

The same searching mechanism is also evident for larger grid sizes with the implicit embryogeny, with similar levels of redundancy observed. However, because the number of rules did not increase much beyond 12, such redundancy becomes a more acceptable compromise for larger problems.

So how does evolution fine-tune solutions to make them match the target letters? Both types of embryogeny seem to begin by filling a large part (or all) of the phenotype grid, and then 'pruning away' unnecessary elements, see figures 5 and 6 . The explicit embryogeny achieves this by pruning branches of its trees; the implicit embryogeny makes use of 'kill' rules to remove elements. Of the two, the implicit embryogeny goes to the furthest extreme with this technique - often by evolving a completely solid block and then picking out the odd element, see figure 6.

The fitness function may be to blame for the "carving letters from a solid block' approach - for it awards considerably higher fitnesses for solid shapes than for emptier ones. 


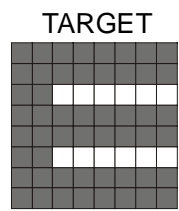

GEN. 30

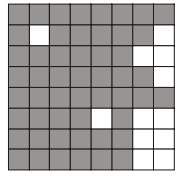

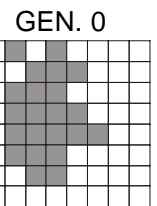

GEN. 40

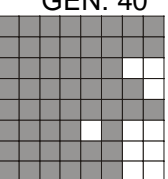

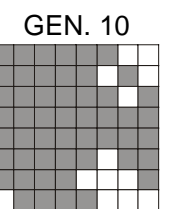

GEN. 50

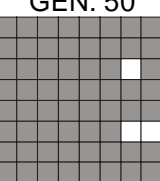

GEN. 20

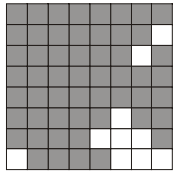

GEN. 100

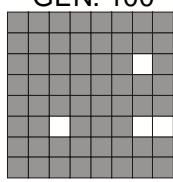

Figure 5: The evolution of an 'E' using the explicit embryogeny. The best new shapes grown in the population are shown every 10 generations (except where no change occurred). The final shape has a fitness of 8 and a soln. size of 251 nodes.

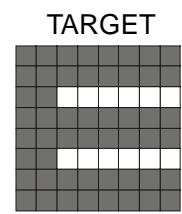

GEN. 30

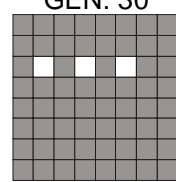

GEN. 80

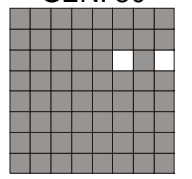

GEN. 0

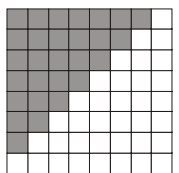

GEN. 40

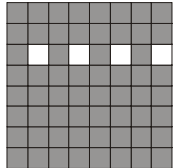

GEN. 90

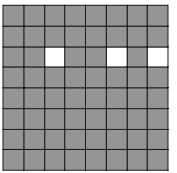

GEN. 10

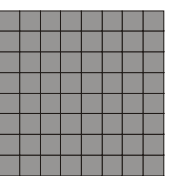

GEN. 60

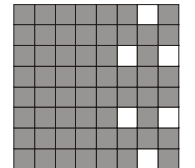

GEN. 100

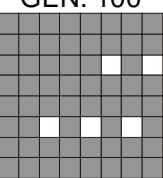

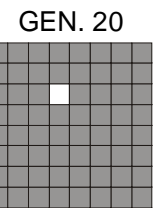

GEN. 70

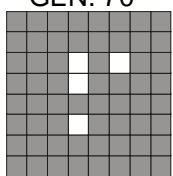

Figure 6: The evolution of an ' $E$ ' using the implicit embryogeny. The best new shapes grown in the population are shown every 10 generations (except where no change occurred). The final shape has a fitness of 7 and a solution size of 11 rules.

As Dawkins (1987) points out, certain embryogenies are better than others at producing certain morphologies. This observation seems to be echoed in this work too. The explicit embryogeny found morphologies such as C's and O's difficult, whereas the implicit was able to handle these morphologies with relative ease. This can be attributed to the fact that the implicit need only generate a few general growth rules for specific directions, and in doing so, can start from a single seed and grow to encompass all four sides of the grid whilst leaving (or killing), for example in the case of the letter ' $\mathrm{O}$ ', a hole in the middle. This is difficult for the explicit embryogeny as it must evolve a long and difficult growth path around the edge of the grid, whilst keeping the centre of the shape free of elements. 
The way in which evolution attempted to generate morphology indicates two points: firstly that the implicit embryogeny seems to have considerable potential because of its impressive scalability, perhaps more so than the explicit embryogeny. Secondly, the representation used for the implicit embryogeny is not as amenable to evolution as we would desire. This may be caused by:

- the representation, which allows dissimilar phenotypes to be close together in the solution-space, providing a discontinuous search-space of solutions and thus causing problems for evolution.

- ineffective positional rules. Although the notion of 'zones' improved the quality of solution for the implicit more than without, it is clear that positional information is hard to glean using the current implicit system, making the evolution of specific rules difficult, leading in turn to bad fitness results.

\section{Biologically plausible implicit embryogeny}

With the results of this experiment in mind, a new implicit model is now under development by the authors. The current system has been extended from the twodimensional cellular automata, to an isospatial grid system. The isospatial grid is a coordinate system developed by Frazer which uses six axis to define a point in space, yielding twelve equidistant neighbours for each point (Frazer, 1995).

The new embryogeny system uses spheres to represent cells and builds threedimensional morphologies by carefully placing and organising a colony of cells using a growth process. This process will use the concept of freely diffusing morphogens to allow cells to acquire positional-information. In addition, key embryological processes such as differentiation and pattern formation shall be investigated to grow morphologies. Although heavily inspired by biology, this system is not intended as a model of biological development. Instead, the work is aimed at extending the capabilities and scalability of evolutionary algorithms.

An implicit embryogeny based system is used to evolve rules that are able to grow designs in complex ways. A chromosome comprises a series of rules (genes). A rule consists of a precondition field and an action field. Each cell has a copy of the chromosome. The rules are applied (expressed) by matching the preconditions to a cell's state. If the preconditions are satisfied the rule is expressed. In this way, rules expressed earlier on in the development can affect other rules by switching them on or off.

Notions of evolvability and speed of growth are playing a key role in the design of this new implicit system. Recent ideas about representations and evolvability such as the use of component based approaches (Bentley, 2000) and neutral networks (Barnett, 1997) may throw light on more suitable representations. The authors are also intending to use a parallel Beowulf supercomputer to exploit the inherent parallelism of growth processes.

Figure 7 shows six morphologies grown from random genomes using the new system. It is clear that the isospatial grid enables surprisingly organic forms to emerge. Shape 4 also illustrates how flat surfaces can arise from multiple cells. 


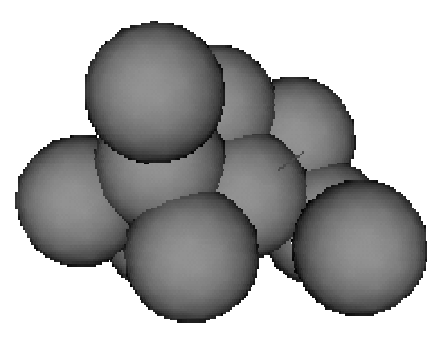

1

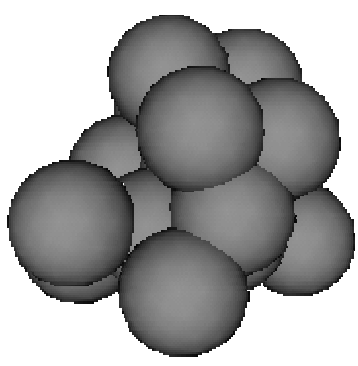

2

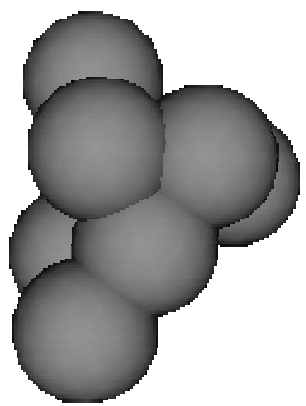

5

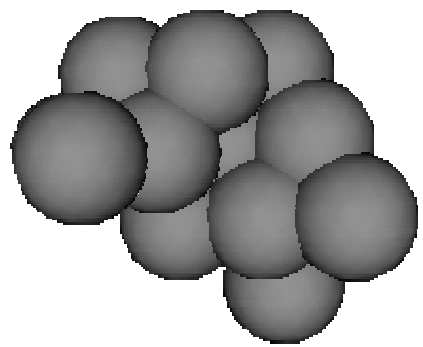

2

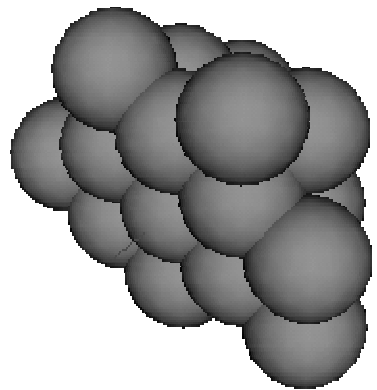

4

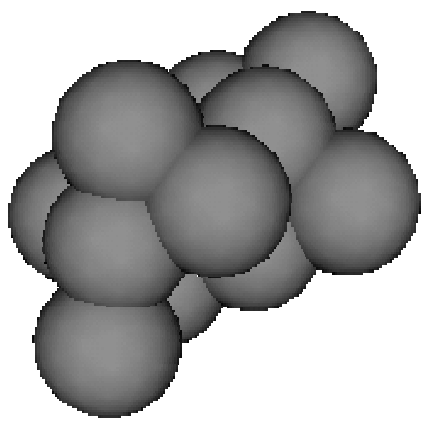

6

Figure 7: Diagram showing six example random morphologies grown by the new system without any morphogens in the environment. 


\section{Conclusions}

This chapter has introduced computational embryology. It began with a historical review of embryology in biology and then described the recent development of computational embryology in computer science. The chapter then looked at present computational embryogenies, comparing the evolvability and scalability of two different approaches (explicit and implicit) for the problem of evolving shape morphologies.

The behavioural analysis of these two embryogenies have shown that both are good at growing different shape morphologies, and that evolutionary computation can benefit from the use of embryogenies.

In addition, this work highlighted some of the problems that require attention with regard to designing evolvable embryogenies. For example, biological concepts such as positional-information, as echoed in this work in the form of zones, can assist in the evolution of shape morphologies, but need to be very carefully designed.

The paper ended with a brief description of a future computational embryogeny system using more biologically plausible methods to overcome the problems encountered in the experiment.

Nature has been successfully evolving complex animals for millions of years. It is the concept of an embryogeny (which itself evolved in nature) that has allowed the evolution of these complex designs.

\section{Acknowledgments}

This work is funded by Science Applications International Corporation (SAIC). Our thanks to the members of nUCLEAR for their useful comments and discussions on this work.

\section{References}

Bard, J. (1990). Morphogenesis. Cambridge University Press, UK.

Barnett, L. (1997). Tangled Webs: Evolutionary Dynamics on Fitness Landscapes with Neutrality. M.Sc. Dissertation. University of Sussex, Brighton, UK.

Bentley, P. J. (2000). Exploring Component-Based Representations - The Secret of Creativity by Evolution. Fourth International Conference on Adaptive Computing in Design and Manufacture (ACDM 2000), University of Plymouth, UK. (To appear).

Bentley, P. J. (Ed.) (1999). Evolutionary Design by Computers. Morgan Kaufman Pub.

Bentley, P. J. (1999). Evolving Fuzzy Detectives: An Investigation into the Evolution of Fuzzy Rules. Chapter in Suzuki, Roy, Ovasks, Furuhashi, and Dote (Eds), Soft Computing in Industrial Applications. Springer Verlag London Ltd.

Bentley, P. J. \& Kumar, S. (1999). Three Ways to Grow Designs: A Comparison of Embryogenies for an Evolutionary Design Problem. In Genetic and Evolutionary Computation Conference (GECCO) Orlando, Florida, USA.(to appear)

Bentley, P. J. \& Wakefield, J. P. (1996). Hierarchical Crossover in Genetic Algorithms. In 
Proceedings of the 1st On-line Workshop on Soft Computing (WSC1), (pp. 37-42), Nagoya University, Japan.

Coates, P., (1997) Using Genetic Programming and L-Systems to explore 3D design worlds. CAAD Futures'97, R. Junge (ed), Kluwer Academic Publishers, Munich.

Dawkins, R. (1987). The Evolution of Evolvability. Proceedings of Artificial Life VI. Langton (Ed.) USA.

de Garis, H. (1999) Artificial Embryology and Cellular Differentiation. Ch. 12 in Bentley, P. J. (Ed.) Evolutionary Design by Computers. Morgan Kaufman Pub.

Frazer, J. (1995). An Evolutionary Architecture. Architecture Association, London.

Goldberg, D.E. Genetic algorithms in search, optimization and machine learning. Addison-Wesley, Reading, MA, 1989.

Holland, J. H. (1975). Adaptation in Natural and Artificial Systems. The University of Michigan Press, Ann Arbor.

Koza, John R. (1992). Genetic Programming I: On the Means of Natural Selection. San Francisco, CA: Morgan Kaufmann.

Koza, John R., Bennett III, Forrest H, Andre, David, and Keane, Martin A. (1999). Genetic Programming III. San Francisco, CA: Morgan Kaufmann.

Kumar, S. (1999) Lessons from Nature: The Benefits of Embryology. (GECCO) Genetic \& Evolutionary Computation Conference. Orlando, Florida, USA.

Kumar, S. \& Bentley, P. J. (1999) The ABCs of Evolutionary Design: Investigating the Evolvability of Embryogenies for Morphogenesis. (GECCO) Genetic \& Evolutionary Computation Conference. Orlando, Florida, USA.

Lawrence, P. A. (1995). The Making of a Fly: The Genetics of Animal Design. Blackwell Science Ltd, The Alden Press, Oxford, UK.

Mallinson, H and Bentley, P. J.. (1999) Evolving Fuzzy Rules for Pattern Classification. In International Conference on Computational Intelligence for Modelling, Control and Automation - CIMCA'99.

N. Jakobi (1995). Harnessing Morphogenesis. International Conference on Information Processing in Cells and Tissues, Liverpool, UK.

Sims, K. (1999). Evolving three-dimensional Morphology and Behaviour. Ch. 13 in Bentley, P. J. (Ed.) Evolutionary Design by Computers. Morgan Kaufman Pub.

Slack, J. M. (1991). From Egg to Embryo. Cambridge University Press.

Turing, A.M. (1952). The chemical basis of morphogenesis. Phil. Trans. R. Soc., 237B, 3772.

Wolpert, L. (1998). Principles of Development. Oxford University Press, Oxford, UK. 